\title{
LETTERS
}

\section{Poincaré plot in obstructive sleep apnoea patients before and after CPAP treatment}

\section{To the Editors:}

Adults with obstructive sleep apnoea (OSA) display substantial heart rate changes during sleep, associated with obstructive respiratory events [1, 2]. These heart rate changes are significantly attenuated or abolished with effective continuous positive airway pressure (CPAP) treatment [3].

Heart rate variability (HRV) refers to the regulation of the sinoatrial node by the sympathetic and parasympathetic branches of the autonomic nervous system. Evaluation of autonomic heart rate control by means of HRV measures has been widely accepted as an important source of information on cardiovascular function [4,5]. HRV is important because it provides a window to observe the heart's ability to respond to normal or abnormal regulatory impulses that affect its rate and rhythm.

Several sophisticated methodologies were developed for measuring HRV, notably those using the heart rate power spectrum and nonlinear dynamics analysis [6]. However, based on the literature, no simple methodology is available for comprehensive use in daily medical practice.

However, a Poincaré or Lorenz plot is a simple semiquantitative method for visualising and calculating HRV [710]. It has been previously shown that the length and width of a Poincaré plot are a weighted combination of low- and highfrequency powers, so it provides a theoretical link between frequency-domain spectral analysis techniques and timedomain Poincaré plot analysis [7].

Despite its advantages, a Poincaré plot has never been used during sleep studies in order to assess HRV or as a marker of continuous positive airway pressure (CPAP) effectiveness in evaluating autonomic heart changes in OSA patients.

The goal of this preliminary study was to propose a simple qualitative and quantitative methodology for evaluation of autonomic heart rate control using heart rate Poincaré plots during wakefulness and in each sleep stage (non-rapid eye movement (NREM) N1-N2, N3 and rapid eye movement sleep), in patients with OSA before and after CPAP treatment.

Our study included 25 patients (18 males and seven females, aged $33.6 \pm 11 \mathrm{yrs}$, body mass index $30 \pm 5.2 \mathrm{~kg} \cdot \mathrm{m}^{-2}$ ) with moderate-severe OSA. Five patients had hypertension (four patients were undergoing treatment with angiotensin converting enzyme (ACE) inhibitor or ACE inhibitor/diuretic combination), two patients were diabetic and one had hypothyroidism. None of the patients had a history of cardiac arrhythmias. Attended overnight polysomnography (Somnostar; Viasys, Hoechberg, Germany) was performed before and after CPAP treatment. During sleep, the apnoea-hypopnoea index, desaturation index and mean oxygen saturation level before and after CPAP treatment were $38.6 \pm 19.4$ versus $2.8 \pm 1.5$ events $\cdot h^{-1}$ $(\mathrm{p}<0.0001), 37.3 \pm 23.9$ versus $2.5 \pm 1.7$ desaturations $\geqslant 4 \%$ per hour $(p<0.0001)$, and $91 \pm 3.6$ versus $94.8 \pm 2.6 \%(p<0.0001)$, respectively. Poincaré plots of sequential R-R intervals (beat to beat variability) were assessed during a 7-h sleep period. We analysed plots both visually and quantitatively using scatter plot length, width and area (calculated from length and width). When estimating the bulk of the plot we excluded obvious isolated outliers, which mainly represent artefacts (fig. 1).

We used the Shapiro-Wilk test to gather evidence about the "non-normality" of the sample. As differences between pairs tended to normality, we used the more sensitive paired t-test to compare the heart rate response before and after CPAP application. One way ANOVA with post hoc Scheffe multiple comparisons was used to evaluate significant differences between Poincaré plot parameters in various sleep stages and wakefulness before CPAP application.

Visual analysis showed a torpedo, fan shaped or complex shaped plots (complex referring to clustering of data points) during sleep stages in OSA patients and mainly comet shaped plots during wakefulness.

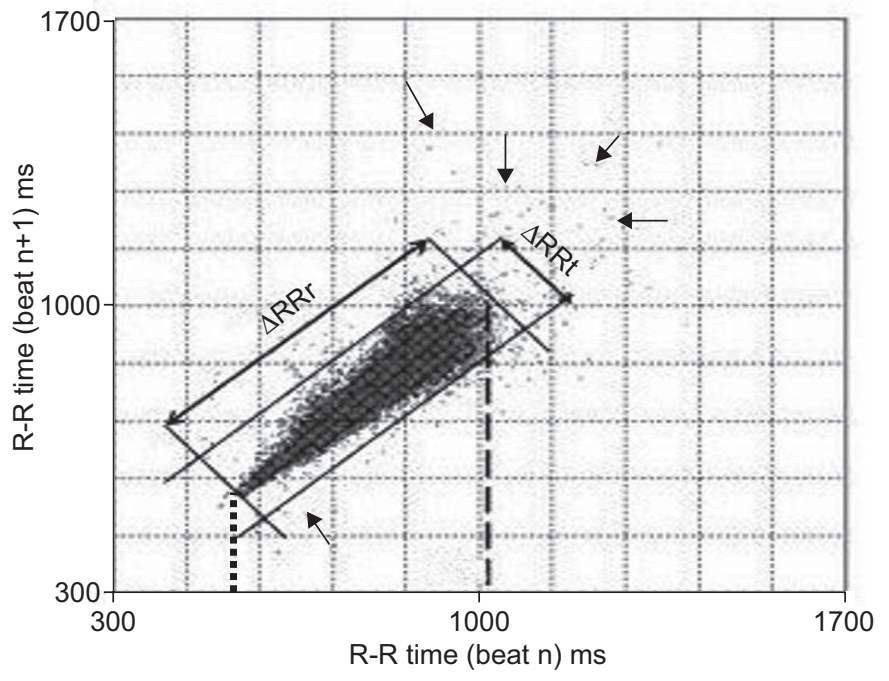

FIGURE 1. Poincaré plot analysis. …... maximal heart rate $(H R)$ frequency (minimal RR interval); - - - -: minimal HR frequency (maximal RR interval); $\Delta \mathrm{RRr}$ difference on plot diagonal between minimal (RRmin) and maximal (RRmax) RR values; $\Delta$ RRt: maximal HR variability as maximal width-difference between of two points at parallel tangential lines determining plot. The square of the plot represents overall HR variability. Isolated outliers (arrows) represent artefacts. 


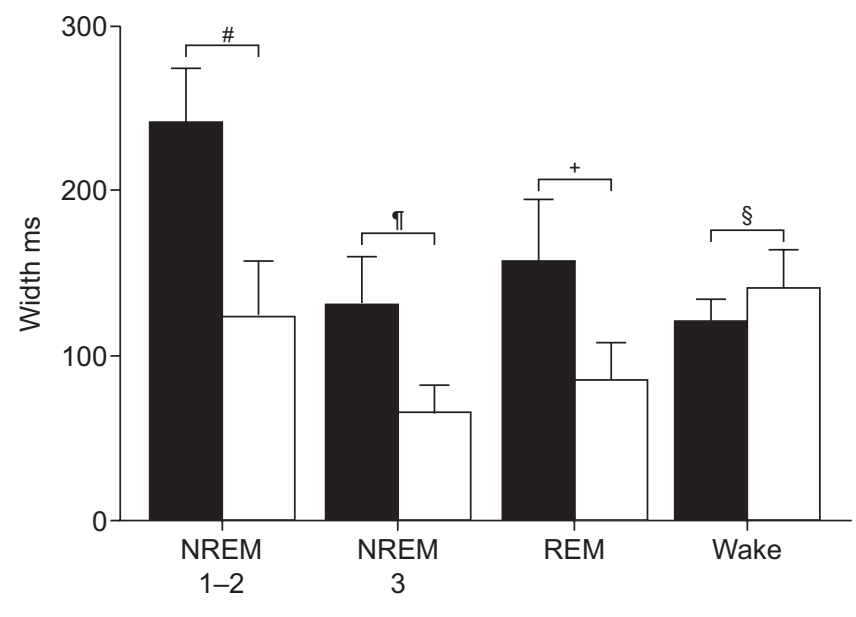

FIGURE 2. Width of Poincaré dispersed cloud (maximal heart rate variability as maximal width-difference between of two points at parallel tangential lines determining plot) in various sleep stages and wakefulness, before (ם) and after $(\square)$ the application of continuous positive airway pressure therapy. NREM: nonrapid eye movement; REM: rapid eye movement. ${ }^{*}: p=0.01 ;{ }^{\bullet}: p=0.049 ;{ }^{+}: p=0.044$; s. $p=0.58$.

$\Delta$ RRt (width of the cloud referring to the maximal heart rate variability) of sleep stages NREM N1-2 was found to be different from $\Delta$ RRt sleep stage N3 $(p=0.0024)$ and from wakefulness $(p=0.0046)$, as well as area plot sleep stage N1-2 versus $\mathrm{N} 3, \mathrm{p}=0.0062$ but not from wakefulness $(\mathrm{p}=0.077)$.

Statistically significant differences were observed in the square of the plot (referring to the overall HRV) in sleep stages NREM N1-2 before and after CPAP therapy $\left(241.7 \mathrm{~ms}^{2} \times 10^{-3} \pm 22.3\right.$ versus $79.8 \mathrm{~ms}^{2} \times 10^{-3} \pm 34.7$, respectively) $(\mathrm{p}=0.001) . \quad \Delta \mathrm{RRt}$ showed statistically significant differences in all sleep stages before and after CPAP therapy (fig. 2). The fact that area plots after CPAP in NREM sleep stages 1-2 (data not shown) were found relatively more decreased in comparison to the respective width of the plot (fig. 2), is indirect evidence of a diminished low frequency/high frequency ratio, i.e. a reduction of sympathetic heart activity particularly evident in NREM sleep stages 1-2 (since the area is the product of length and width).

Our study is the first to use the Poincaré plot method in patients with OSA before and after CPAP treatment. Autonomic disorganisation due to repetitive bradytachycardia events, as a result of obstructive events, is a common feature in OSA patients and the resolution of this phenomenon is a marker of effective CPAP treatment. HRV data were used in research studies, although not in daily clinical practice due to the complexity of most of the methods used. Poincaré plot analysis is a simple and possibly superior methodology compared to the classical methods that assess HRV. The square of the plot representing the overall HRV is easy to calculate and does not need special knowledge or training. This method might be applied to clinical practice in OSA patients for purposes such as evaluation of abnormalities in autonomic control or as a practical way to assess the effectiveness of CPAP treatment more specifically as a marker of sympathetic activity reduction. As HRV and the autonomic heart response are an important issue in OSA patients, the influence of CPAP therapy needs to be further assessed.

\section{Mermigkis, D. Mermigkis, G. Varouchakis, S. Schiza and P. Panagou}

Sleep Disorders Center, General Army Hospital, Athens, Greece.

Correspondence: C. Mermigkis, Thrakis 61A, Brilissia, Athens, Greece. E-mail: mermigh@gmail.com

Statement of Interest: None declared.

\section{REFERENCES}

1 Narkiewicz K, Montano N, Cogliati C, et al. Altered cardiovascular variability in obstructive sleep apnea. Circulation 1998; 98: 1071-1077.

2 Wiklund U, Olofsson BO, Franklin K, et al. Autonomic cardiovascular regulation in patients with obstructive sleep apnoea: a study based on spectral analysis of heart rate variability. Clin Physiol 2000; 20: 234-241.

3 Khoo MC, Belozeroff V, Berry RB, et al. Cardiac autonomic control in obstructive sleep apnea: effects of long-term CPAP therapy. Am J Respir Crit Care Med 2001; 164: 807-812.

4 La Rovere MT, Bigger JT, Marcus FI. Baroreflex and heart rate variability inprediction of total cardiac mortality after myocardial infarction. Lancet 1998; 14: 478-484.

5 Raetz SL, Richard C, Garfinkel A, et al. Dynamic characteristic of cardiac R-R intervals during sleep and waking states. Sleep 1991; 14: 526-533.

6 Heart rate variability, standards of measurement, physiological interpretation and clinical use. Task Force of the European Society of Cardiology and the North American Society of Pacing and Electrophysiology. Circulation 1996; 93: 1043-1065.

7 Hnatkova K, Copie X, Staunton A, et al. Numeric processing of Lorenz plots of R-R intervals from long-term ECG's: comparison with time-domain measures of heart rate variability for risk stratification after myocardial infarction. J Electrocardiography 1995; 28: 74-80.

8 Žemaityte D, Varoneckas G, Ožeraitis E, et al. Autonomic heart rate control evaluation by means of heart rate poincaré plot analysis. Biomedicine 2001; 1: 33-41.

9 Mourot L, Bouhaddi M, Perrey S, et al. Quantitative Poincaré plot analysis of heart rate variability: effect of endurance training. Eur $J$ Appl Physiol 2004; 91: 79-87.

10 Kamen PW, Krum H, Tonkin A. Poincaré plot of heart rate variability allows quantitative display of parasympathetic nervous activity in humans. Clin Sci 1996; 91: 201-208.

DOI: $10.1183 / 09031936.00061609$ 